University of Nebraska - Lincoln

DigitalCommons@University of Nebraska - Lincoln

Virology Papers

Virology, Nebraska Center for

2002

\title{
Phylogenetic and Phenotypic Analysis of HIV Type 1 Env gp120 in Cases of Subtype C Mother-to-Child Transmission
}

\author{
Hong Zhang \\ University of Nebraska-Lincoln \\ Guillermo Ortí \\ University of Nebraska-Lincoln \\ Qiujiang Du \\ University of Nebraska-Lincoln \\ Jun $\mathrm{He}$ \\ University of Nebraska-Lincoln \\ Chipepo Kankasa \\ University Teaching Hospital (Lusaka, Zambia) \\ See next page for additional authors
}

Follow this and additional works at: https://digitalcommons.unl.edu/virologypub

Part of the Virology Commons

Zhang, Hong; Ortí, Guillermo; Du, Qiujiang; He, Jun; Kankasa, Chipepo; Bhat, Ganapati; and Wood, Charles, "Phylogenetic and Phenotypic Analysis of HIV Type 1 Env gp120 in Cases of Subtype C Mother-to-Child Transmission" (2002). Virology Papers. 229.

https://digitalcommons.unl.edu/virologypub/229

This Article is brought to you for free and open access by the Virology, Nebraska Center for at DigitalCommons@University of Nebraska - Lincoln. It has been accepted for inclusion in Virology Papers by an authorized administrator of DigitalCommons@University of Nebraska - Lincoln. 


\section{Authors}

Hong Zhang, Guillermo Ortí, Qiujiang Du, Jun He, Chipepo Kankasa, Ganapati Bhat, and Charles Wood 


\title{
Sequence Note
}

\section{Phylogenetic and Phenotypic Analysis of HIV Type 1 Env gp120 in Cases of Subtype C Mother-to-Child Transmission}

\author{
HONG ZHANG, ${ }^{1,2}$ GUILLERMO ORTÍ, ${ }^{2}$ QIUJIANG DU, $, 1,2$ JUN HE, ${ }^{1,2}$ CHIPEPO KANKASA, ${ }^{3}$ \\ GANAPATI BHAT, ${ }^{3}$ and CHARLES WOOD ${ }^{1,2}$
}

\begin{abstract}
Human immunodeficiency virus type 1 (HIV-1) subtype C constitutes a majority of the newly transmitted cases of HIV-1 in many developing countries. There is growing evidence suggesting that subtype $C$ viruses may display characteristics that make them distinct from subtype $B$ and other subtypes, and such differences may affect its transmission and pathogenesis in infected individuals. In this study, HIV-1 sequences from the C2-V4 region of the envelope gene were analyzed from four infected mother-infant pairs (MIPs) after perinatal transmission in Zambia. We found that all viral isolates from four Zambian MIPs were of the subtype C HIV-1 subgroup. All tested isolates were macrophage tropic and did not infect any tested $T$ cell lines or form syncytia in vitro. In addition, the isolates appear to use exclusively the CCR5 coreceptor. Phylogenetic analyses clearly indicated two contrasting phylogenetic transmission patterns for the four MIPs analyzed in the present study. Three cases displayed a pattern of selective transmission of a single or a few variants, and one case was found to transmit multiple maternal HIV-1 variants to her infant. The differences in the mode and timing of transmission may account for the observed transmission pattern among four MIPs.
\end{abstract}

$\mathbf{P}$ ERINATAL HIV-1 TRANSMission is a complex multifactorial process that remains poorly understood. Transmission may occur in utero, intrapartum, or postpartum through breast-feeding. Much progress has been made in discerning the virological and immunological factors that are associated with an increased risk of perinatal transmission. These factors include, but are not restricted to, maternal virus load, disease status, $\mathrm{CD}^{+}{ }^{+} \mathrm{T}$ cell count, and HIV-1 env gene diversity. ${ }^{1}$ Information about the molecular mechanisms underlying HIV-1 perinatal transmission has come predominantly from investigations of HIV-1 subtype B. Several studies have shown that only a few genotypes of the maternal virus were transmitted to infants, ${ }^{2}$ suggesting that selection for specific variants of HIV-1 can occur during transmission. Conversely, transmission of multiple HIV-1 genotypes from mothers to infants also has been reported. ${ }^{3}$ Still other studies have demonstrated that both se- lective transmission of a single maternal variant and transmission of multiple variants may occur during perinatal transmission. ${ }^{1,4,5}$ These seemingly discordant observations could be due to differences in the timing of transmission or to differences in the HIV-1 strains and infected populations during perinetal transmission, or could be related to the immunological status of the mothers. Subtype C HIV-1 viruses, initially a rarely reported subtype, are now dominating the global pandemic and constitute a majority of the newly transmitted cases of HIV-1 in many developing countries. It is not clear whether this rapid spread of subtype $\mathrm{C}$ is due to the introduction of a novel subtype into a fertile naive population or whether this subtype may have a higher replication and transmission fitness. There is growing evidence suggesting that subtype $\mathrm{C}$ viruses may display characteristics that make them distinct from subtype B and other subtypes, and that such differences may affect transmis-

\footnotetext{
${ }^{1}$ Nebraska Center for Virology, University of Nebraska, Lincoln, Nebraska 68588.

${ }^{2}$ School of Biological Sciences, University of Nebraska, Lincoln, Nebraska 68588.

${ }^{3}$ Department of Pediatrics, University Teaching Hospital, Lusaka, Zambia.
} 
sion and pathogenesis. ${ }^{6}$ In this article we report the mother-tochild transmission (MTCT) patterns of HIV-1 subtype C in Zambia, in the absence of selective pressure from antiretroviral therapy. A fundamental understanding of the factors that directly correlate with HIV-1 MTCT should reveal the mechanisms involved. Furthermore, a detailed understanding of the mechanisms of MTCT would allow for the development of better strategies for treatment and prevention.

Patient population and the characteristics of their HIV-1 isolates. Four mother-infant pairs (MIPs) labeled 902, 1084, 1288, and 1751 were characterized in this study, including two male and two female infants. The mothers were between 18 and 28 years of age; they were known to be HIV-1 positive at the time of delivery and are likely to have acquired HIV-1 infection heterosexually. All subjects were asymptomatic at the time of delivery without any clinical signs of immunosuppression. The babies were all breast-fed and were followed up at the clinic at 2 (MIP 1751), 4 (MIP 1084), or 6 (MIPs 902 and 1288) months after delivery. Venous blood samples from the mothers were collected before delivery and infant samples were drawn within $24 \mathrm{hr}$ of delivery, if possible. Follow-up blood specimens were obtained from mothers and infants when they came back for follow-up visits 2, 4, and 6 months after delivery. The presence of maternal HIV-1 antibodies was determined by two rapid assays, Capillus (Cambridge Biotech, Ireland) and Determine (Abbott Laboratories, Abbott Park, IL), performed on blood taken at delivery. The positive samples were further confirmed by immunofluorescence assay (IFA). The status of HIV-1 infection in the infants was determined by isolating virus from the infant peripheral blood mononuclear cells (PBMCs) and/or by polymerase chain reaction (PCR) whenever possible. Viruses were isolated from all MIP follow-up samples except for mother 1288. Nevertheless, HIV-1 was detected by PCR from her PBMC DNA. It is likely that mother 1288 transmitted HIV-1 to her infant in utero because HIV-1 sequences were detected by PCR in her cord blood (taken at birth). In contrast, cord blood and infant PBMC DNA were HIV-1 negative by PCR for infants 902, 1084, and 1751 at birth, suggesting that these infants have acquired HIV-1 infection intrapartum or postpartum.

Because infectious HIV-1 was recovered from the PBMCs of all but one (1288M) Zambian mothers, cell tropism and core- ceptor usage of these viral isolates were studied. All viruses replicated to high viral titers, as measured by $\mathrm{p} 24$ production in normal PBMCs and monocyte-derived macrophages (MDMs), but did not grow in several $\mathrm{T}$ cell lines tested, including MT-2 and C8166. In addition, these viral isolates did not induce syncytia in infected cells in culture (Table 1). These results suggest that the viral isolates from the MIPs are all of the non-syncytium-inducing (NSI) phenotype and cannot grow in established $\mathrm{T}$ cell lines. To confirm their macrophage tropism in culture, coreceptor usage also was evaluated with cell lines that specifically express different coreceptors. None of the isolates tested infected CEMx174-GFP cells, which express only CD4 and CXCR4 (gift from W. Xu, Harvard Medical School, Boston, MA). Similarly, none of the viruses tested grew in cells expressing CCR3 and CD4 (HOS-CD4-CCR3) or in CCR5- 32 PBMCs (gift from J. Hoxie, University of Pennsylvania). In contrast, cells expressing CCR5 and CD4 (GHOST-CD4-CCR5) (gift from W. Xu, Harvard Medical School) were readily infected with all tested isolates, suggesting that the MIP HIV-1 isolates use CCR5 but not CCR3 or CXCR4 as coreceptor (Table 1). This is in agreement with the infectivity assays demonstrating that only primary PBMCs and MDMs supported viral growth. These findings are consistent with reports demonstrating that HIV-1 subtype $\mathrm{C}$ is distinct from the other subtypes, such as subtype B, in the evolution of its coreceptor utilization with rare CXCR4 usage even when viral isolates were obtained from late-stage AIDS patients.?

Sequence variation at the env gene. To determine HIV-1 env gene sequence variation of the MIP HIV-1 samples, PCR analysis was carried out. Primer design was based on a reference alignment of all HIV-1 subtypes obtained from the Los Alamos database (http://hiv-web.lanl.gov).Concerning the sample from mother 1288 (1288M), we failed to isolate virus from this subject and the template DNA was extracted directly from uncultured patient PBMCs. For the other specimens, only cultured viruses, but no residual patient PBMCs, were available for PCR analysis. The amplified products were cloned and a representative measurement of genetic variation for the viral populations was obtained by screening 30-45 clones for each sample by the single-strand conformational polymorphism (SSCP) as previously described..$^{8}$ The 491 -bp colony PCR products encoding the V3 loop and flanking regions of the env gene were

Table 1. Viral Phenotype and Chemokine Coreceptor Usage

\begin{tabular}{|c|c|c|c|c|c|c|c|c|c|c|}
\hline \multirow[b]{2}{*}{ Patient } & \multirow{2}{*}{$\begin{array}{c}\text { Sampling } \\
\text { time }^{\mathrm{a}} \\
\text { (month) }\end{array}$} & \multirow[b]{2}{*}{ Phenotype } & \multicolumn{2}{|c|}{ PBMCs } & \multicolumn{3}{|c|}{ Tropism } & \multicolumn{3}{|c|}{ Coreceptor usage } \\
\hline & & & $C C R 5^{+}$ & $C C R 5-\Delta 32$ & Macrophage & $M T-2$ & $C 8166$ & CCR3 & CXCR4 & CCR5 \\
\hline $1751 \mathrm{M}^{\mathrm{b}}$ & 2 & NSI & + & - & + & - & - & - & - & + \\
\hline $1751 \mathrm{I}^{\mathrm{c}}$ & 2 & NSI & + & - & + & - & - & - & - & + \\
\hline $1084 \mathrm{M}$ & 4 & NSI & + & - & + & - & - & - & - & + \\
\hline 1084I & 4 & NSI & + & - & + & - & - & - & - & + \\
\hline $902 \mathrm{M}$ & 6 & NSI & + & - & + & - & - & - & - & + \\
\hline $902 \mathrm{I}$ & 6 & NSI & + & - & + & - & - & - & - & + \\
\hline $1288 \mathrm{I}$ & 6 & NSI & + & - & + & - & - & - & - & + \\
\hline
\end{tabular}

asampling time in months after delivery, when each blood sample was taken.

${ }^{\mathrm{b}} \mathrm{M}$, Mother.

${ }^{\mathrm{c}} \mathrm{I}$, Infant. 
amplified for SSCP analysis by using primers LKF (5'-CCTGCTGGTTATGCGATTCTAAA; corresponding to positions 6882-6904 of the HIV-1 HxB2 strain) and LKR (5' -CAATAGAAAAATTCTCCTCTACAATTAAA; complementary to positions 7378-7350). Clones demonstrating diversity by SSCP were used for sequence analysis. A 617-bp fragment spanning the $\mathrm{C} 2-\mathrm{V} 4$ region was amplified with primers LKF and LKS (5'-ACCTCCTGCCACATGTTTATAATTTG; complementary to positions 7513-7488) and sequenced. A total of 25-40 clones was sequenced in both directions for each sample to obtain a representative measurement of the diversity of the viral genotypes.

All sequences obtained were scanned for open reading frames and excluded from the analysis if the reading frame was interrupted. For the four MIPs a total of 139 distinct genotypes with uninterrupted reading frame were characterized. For most MIPs, the number of HIV-1 env genotypes detected in mothers and their infants was similar, with the exception of MIP 1288
(22 genotypes detected for the mother vs. 15 for the infant). As described above, $1288 \mathrm{M}$ env sequences were amplified by PCR directly from PBMC DNA, and not from cocultured cells as for all other samples. To gauge the level of variation for viral populations in each patient, nucleotide diversity $(\pi)$ and genetic diversity (the parameter $\theta$ ) were estimated with the program UPBLUE. ${ }^{9}$ Nucleotide diversity values measure the average number of substitutions between two sequences and $\theta=2 \mathrm{Ne} \mu$, where $\mathrm{Ne}$ is the inbreeding effective population size and $\mu$ is the per-nucleotide mutation rate. Again, with the exception of MIP 1288, no major differences between maternal and infant sequences for each MIP were observed for this parameter (Fig. 3 ). However, some variation was observed among MIPs. Because of the presence of a divergent set of HIV-1 genotypes isolated from MIP 1084, more frequent in mother 1084 than in her infant, the nucleotide diversity value $(\pi=20.4)$ is higher in this case than in all other MIPs (typically ranging between 4 and 13). The genetic diversity parameter $\theta$ showed maximal

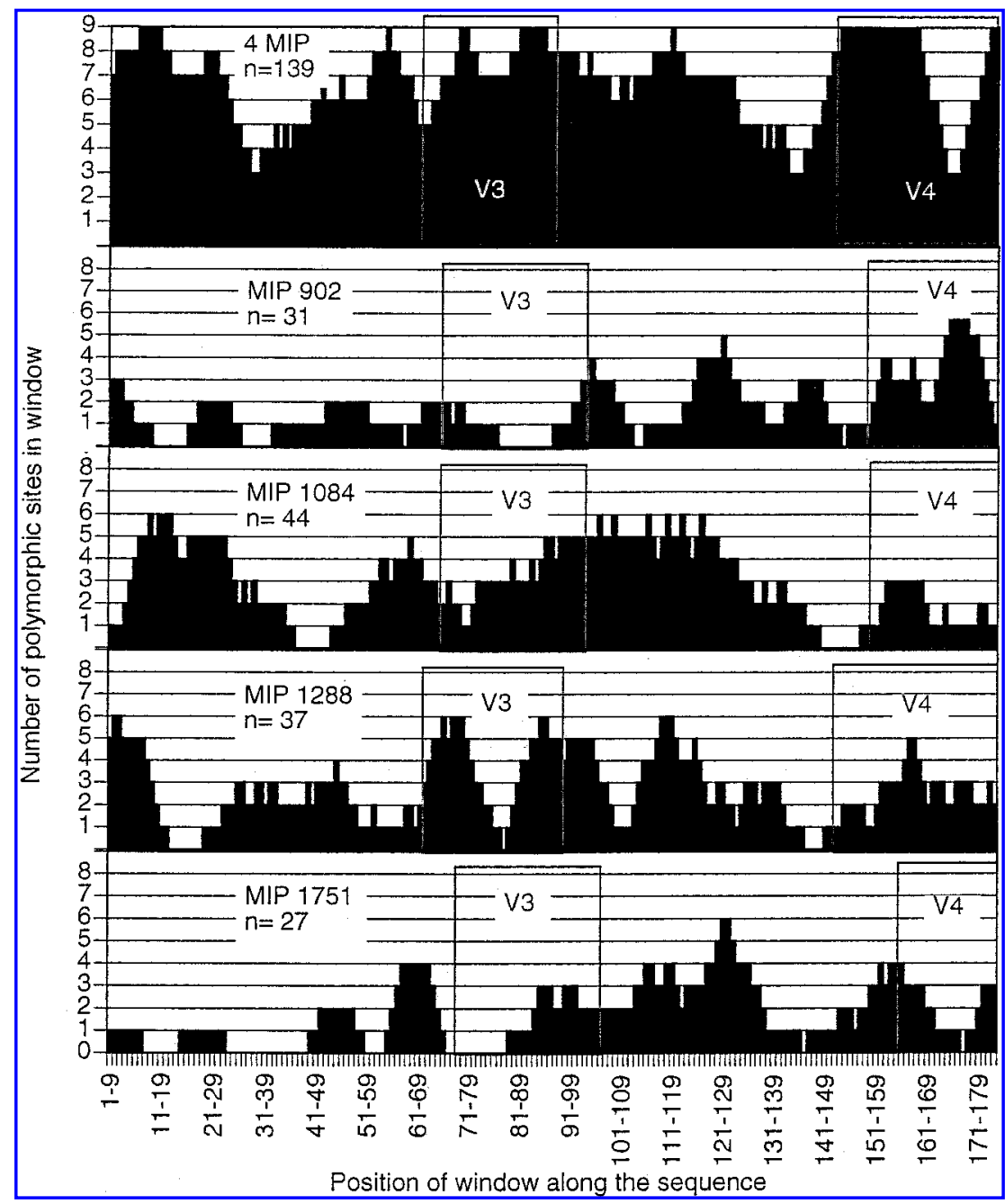

FIG. 1. Sliding-window analysis of sequence variation in the $\mathrm{C} 2-\mathrm{V} 4$ regions at the protein level. The shaded area indicates the number of variable sites in a window of nine residues along the protein sequence. Top panel (4MIP) is from an alignment of all 139 HIV-1 env genotypes characterized in this study. Lower panels summarize variation among genotypes characterized from each MIP. The V3 and V4 loops are boxed, and their relative positions among MIPs differ because of insertions and deletions of residues in the $\mathrm{C} 3$ and $\mathrm{V} 4$ regions. 


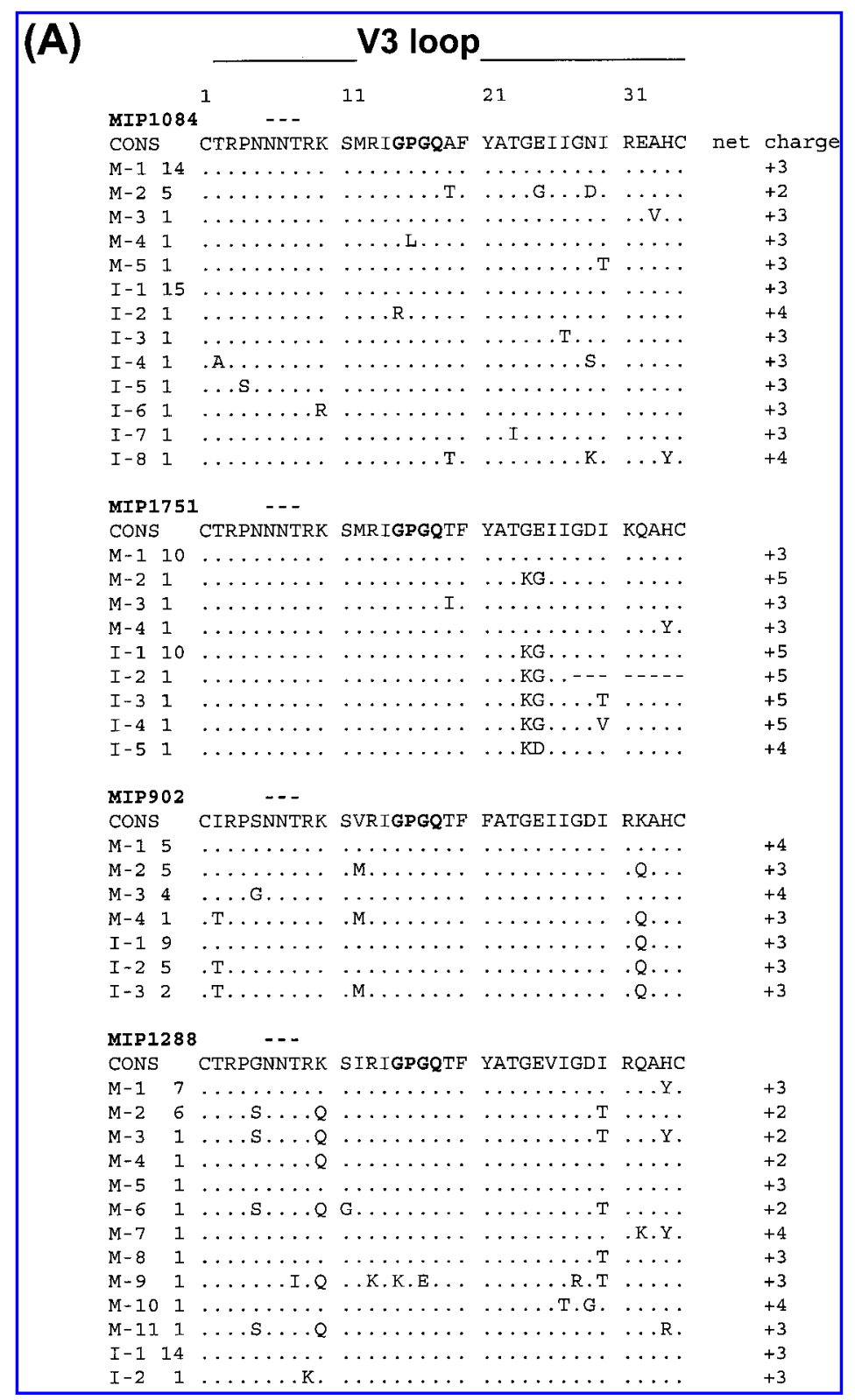

FIG. 2. Multiple alignment of deduced amino acid sequences of the V3 loop (A) and V4 loop (B) derived from the four MIPs. Sequences from each pair are aligned against the consensus sequence (CONS) derived from each mother's sequences in each region. The frequency of clones with identical amino acid sequences for each sample is given at the end of the sequence. Dots indicate identity with the reference sequence, dashes represent gas introduced to maximize alignment. Potential N-linked glycosylation sites are indicated by three continuous dashes above the alignment. The tetrameric motif GPGQ in V3 is in boldface. Amino acid positions above the alignment are numbered according to their positions in the V3 or V4 loop. Net charges in V3 were calculated by the number of positively charged amino acids ( $\mathrm{R}$ and $\mathrm{K}$ ) minus the number of negatively charged amino acids (D and $\mathrm{E}$ ).

difference again between mother and infant in MIP 1288 (from 7 to 48 , respectively), but for all other cases the range of $\theta$ values among MIPs and between mothers and their infants was not so pronounced (ranging between 11 and 24). A comparison among all viral populations revealed that mother 1288 exhibited the most heterogeneousviral quasi-species. The most likely explanation is that these sequences were directly generated from uncultured PBMCs, whereas sequences of all other samples were obtained from viral cocultures. Selective effects due to viral cultures for a particular strain have been suggested by other studies, which showed that certain HIV-1 variants present in PBMCs in vivo were selected during isolation. ${ }^{10}$

To obtain an estimate of genetic variation in each domain (C2, V3, C3, and V4) of the env gene fragment, a sliding window analysis was performed on the complete 139-sequence alignment and on each of the MIP data sets, using the program 


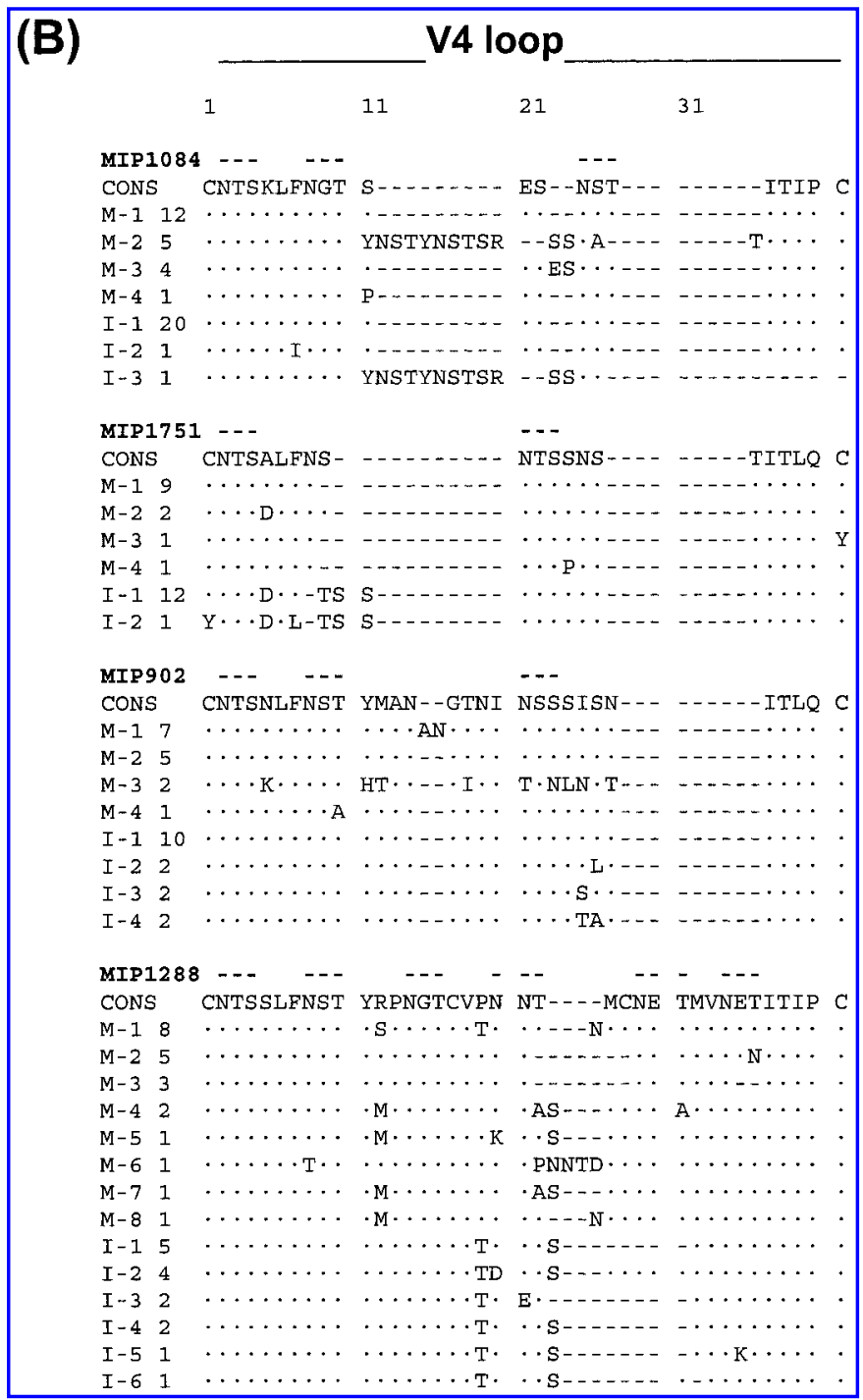

FIG. 2. (continued)

MEGA. ${ }^{11}$ This analysis shows the number of polymorphic sites in an overlapping window of nine amino acids, applied to each of the protein sequence alignments (Fig. 1). A consistent feature of this analysis is that for most MIPs, higher levels of variation were observed in the $\mathrm{C} 3$ and $\mathrm{V} 4$ regions than in the $\mathrm{V} 3$ loop, and length variation also was more frequently encountered in V4. However, for MIPs 1084 and 1288, similar levels of variation were exhibited among these regions. The most common amino acid motifs in V3 and V4 for each MIP are shown in Fig. 2 and are described below.

Correlation between viral phenotype and the molecularfeatures of the env $V 3$ region. The overall charge of the V3 loop has been suggested to influence both cellular and coreceptor tropism of HIV. ${ }^{12,13}$ In addition, the amino acid residues at positions 11,25 , and 28 have been documented to determine macrophage or $\mathrm{T}$ cell line tropism. ${ }^{14,15}$ The HIV-1 sequences obtained form all four MIPs indeed supported the phenotype of the viruses. On the basis of the absence of positively charged amino acids at positions 11,25 , and 28 of the V3 loop, and the overall net charge of the env V3 loop (ranging from +2 to +5 ) (Fig. 2A), viruses isolated from all MIPs would be predicted to be NSI and exhibit CCR5 coreceptor usage, ${ }^{14,16}$ which was confirmed by their in vitro growth properties. The V3 crown motif (positions 15-18) constitutes a major neutralizing epitope and displays extensive variation within the subtype B viruses, with GPGR as the major tetrameric motif. However, in the subtype $C$ sequences analyzed here, the GPGQ tetrapeptide crown motif was present in $98 \%$ (136 of 139) of the V3 loop sequences evaluated in this study. This tetrameric GPGQ motif in the V3 domain has in fact been described as a predominant feature in other HIV1 subtype $\mathrm{C}$ viruses. Furthermore, the potential N-linked glycosylation site sequence NXT or NXS at positions 6-8 of the V3 loop (NNT), which is highly conserved in most HIV-1 isolates, was present in all infant sequences and the majority of mother sequences; only one mother sequence lost this glycosylation site, 1288M-9 (NNI) (Fig. 2A). 
Comparison of the env V4 region amino acid sequences. Previous studies of subtype $C$ sequences have identified more variation in the V4 loop than in V3. ${ }^{17}$ A striking feature of the V4 region observed in this study, for both mother and infant sequences, was length polymorphism, resulting from deletions and insertions (Fig. 2B). Thus, deletions also appear to be a feature contributing to V4 variability, although the outcome and effect of such variation on the subtype $C$ viral phenotype is not known. The longest amino acid sequences were detected in several clones from MIP 1288; the sequences obtained from MIPs 1751 and 1084 were shorter. Furthermore, the deletion and insertion patterns in V4 were congruent within each MIP, but differed among MIPs, indicating characteristic MIP length variation patterns, and in agreement with phylogenetic analyses showing that each MIP forms a distinct and well-supported clade (data not shown). Determination of whether the deletions and insertions in V4 observed in the four Zambian HIV-1-infected MIPs reflect a unique property of subtype $\mathrm{C}$ infection and vertical transmission, or whether the V4 domain of subtype $\mathrm{C}$ viruses could be under immunological or replicative selective pressure, is a focus of ongoing investigations in our laboratory.

Growing evidence has demonstrated a role for N-linked glycans in limiting the recognition of gp120 protein sequences by antiviral antibodies. ${ }^{18}$ In our analyzed clones, deletions of potential N-linked glycosylation sites were commonly observed at several polymorphic sites (Fig. 2B). The first site (NTS) at positions 2-4 of the V4 loop was perfectly conserved in all four MIPs, indicating a possible functional significance for this site. In addition, there are several potential glycosylation sites downstream, but they varied in both number and position among the MIPs. Among them, MIP 1288 had more additional potential glycosylation sites than other MIPs (Fig. 2B). Given the notable differences in the V4 region seen among mother 1288 and other MIP sequences (Fig. 2B), it is possible that the selective effect may involve length polymorphism and $\mathrm{N}$-glycosylation site variations in this region.

Phylogenetic analysis. To identify the subtype affiliation, phylogenetic analyses were performed on two representativesequences per MIP (617 bp, C2-V4) with a panel of reference sequences obtained from the HIV Sequence Database (http://hiv-web.lanl.gov). All nucleotide sequences were translated and the amino acid sequences were aligned, using CLUSTAL X. ${ }^{19}$ The protein sequence alignment obtained was used to manually align the nucleotide sequences with the SeA1 sequence editor to preserve the reading frame (i.e., no alignment gaps were introduced within codons). This procedure was followed for all nucleotide alignments analyzed in this study. The alignment containing the MIP and subtype reference sequences (50 sequences in total, $640 \mathrm{bp}$ ) was analyzed on the basis of the minimum evolution (ME) criterion, using PAUP* version $4.0 \mathrm{~b} 8 .^{20}$ Heuristic searches used the TBR branch-swapping algorithm for 10 replications on initial trees obtained by stepwise addition with random addition order. Distance measures were based on the GTR $+\mathrm{I}+\mathrm{G}$ model selected by MODELTEST, ${ }^{21}$ using pairwise deletion of alignment gaps. Bootstrap analysis with 100 pseudoreplications was used to test robustness of the phylogenetic results. All Zambian MIP viral sequences characterized in this study grouped unambiguously with the reference subtype C sequences with high (98\%) bootstrap support (results not shown). After confirmation of subtype affiliation, all nucleotide sequences ( $617 \mathrm{bp}$ ) obtained from the four MIPs, representing a total of 139 sequences, were aligned as described above and compiled into a single file for phylogenetic analysis by PAUP*. The alignment, containing a total of 139 sequences, was analyzed by the ME method, with a simplified heuristic search with the SPR branch-swapping algorithm and by bootstrap analysis with the neighbor-joining (NJ) algorithm. Genetic distances among DNA sequences were estimated with the K81uf $+\mathrm{G}$ model, selected with the program MODELTEST. Results from this phylogenetic analysis were used to test the vertical transmission hypothesis for each MIP. All trees obtained support the monophyletic grouping of all mother and infant sequences isolated for each MIP. This result was supported by bootstrap analysis using the neighbor-joining (NJ) method (100\% support for all branches grouping each MIP sequence pair; data not shown). The strong support for grouping all sequences from each MIP unambiguously indicates vertical transmission of HIV from mother to infant in all cases.

A more thorough phylogenetic analysis strategy was used to analyze each set of MIP viral sequences separately, using three sequences of other MIPs as outgroups (to root the phylogeny). Because all phylogenetic methods assume that no recombination has occurred among the sequences compared, the first step in this analysis involved testing for recombination among potentially recombining HIV-1 sequences for each MIP data set. The informative-sites test $\mathrm{t}^{22}$ was used to test the hypothesis of clonality, as implemented in the program PIST. ${ }^{23}$ No significant score $(p<0.05)$ was obtained, indicating that recombination among maternal or infant viral env sequences was not detected by this method.

For each MIP data set, MODELTEST was used to determine a justified model of evolution and this model was applied to calculate genetic distances for phylogenetic analysis. All phylogenetic inference used the ME method. Heuristic searches were based on 20 replicates with starting trees obtained via stepwise addition (with random addition order for each replicate). All optimal trees were saved. Bootstrap analysis with 200 pseudoreplications was used to assess support of the resulting topology. Each pseudoreplicate was analyzed by a complete heuristic search with SPR branch swapping on a single starting tree obtained by neighbor joining $(\mathrm{NJ})$. The main results of these analyses are shown in Fig. 3. Two major patterns emerged. For MIPs 1288, 1751, and 902, all infant sequences formed a wellsupported monophyletic group, either nested within the mother's sequences (MIPs 1751 and 902) or forming their sister group (MIP 1288). Bootstrap support for this pattern was high, ranging from $95 \%$ for MIP 1288 to $61 \%$ for MIP 902 (Fig. 3, see numbers on branches). Sequences forming the infant clades are consistently genetically less diverse than their maternal counterpart, but this difference is relatively small except for MIP 1288. Mother 1288 displayed much higher viral diversity than her infant. It is likely that several factors could influence the outcome of vertical transmission. One factor is timing of infection, and this may explain the phylogenetic pattern observed for one of the infants (1288) that was infected in utero, because the cord blood taken at birth was found to be HIV-1 positive by PCR. Six months after birth, all viral sequences recovered from infant 1288 clustered tightly $(95 \%$ 


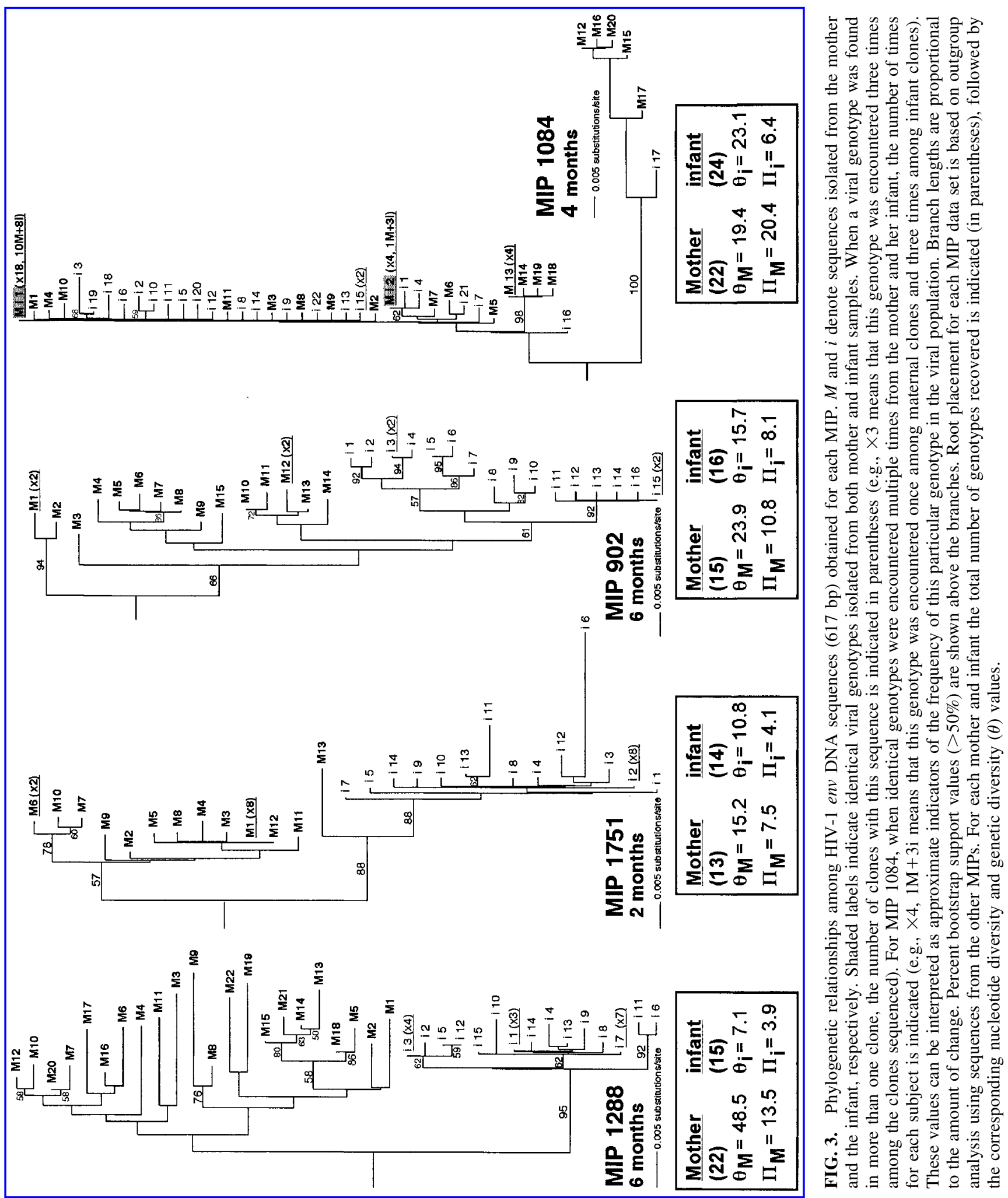


bootstrap support), indicating either selective transmission in utero or rapid lineage sorting after infection, leading to loss of many viral genotypes due to selection. A similar phylogenetic pattern was observed in two other MIPs (902 and 1751), whose infants did not test positive at birth, suggesting later transmission, either during or after birth. Because the timing is unknown either scenario would apply for these cases (selective transmission or massive transmission followed by lineage sorting). The pattern obtained in these three MIPs implies either a scenario of selective transmission of a few viral genotypes from mother to infant or, alternatively, a great loss of viral genotypes in the infant after transmission of multiple variants from the mother.

A contrasting phylogenetic pattern was obtained for MIP 1084. In this case, viral populations isolated from mother and infant samples share a large proportion of their genetic diversity and they do not form separate clades. In fact, in this case two identical genotypes were shared between maternal and infant viral populations (Fig. 3). Furthermore, many equally optimal trees were found, reflecting the presence of many intermediate genotypes that differ only by a few base pairs and give rise to polytomies in the inferred trees. In fact, with the exception of a divergent clade with mother and infant sequences (M12, M15-17, M20, and 117) and a few other nodes, almost no well-supported clades emerge in this tree (see the low bootstrap support values obtained, Fig. 3, MIP 1084). A likely explanation for this pattern is recent (no time for lineage sorting to operate) or ongoing massive transmission (perhaps through breast milk), because all infants continue to be exposed to HIV1 through breast-feeding in addition to the in utero or intrapartum transmitted viruses. Taken together, these results imply that either selective transmission of a single or a few variants, or transmission of multiple maternal HIV-1 variants, could occur with subtype $\mathrm{C}$, regardless of the timing of transmission, which is in agreement with several cases reported for subtype $\mathrm{B}$ and for some non-subtype B HIV-1.4,5,24

In conclusion, we have observed that all viral isolates from four Zambian mother-infant pairs were of the subtype C HIV1 subgroup. All tested isolates were macrophage tropic in culture and used CCR5 as a coreceptor. The transmission of both a selected single maternal variant and multiple maternal variants was observed. Differences in the mode and timing of transmission may contribute to the observed transmission patterns among the four mother-infant pairs. This article extends our knowledge of subtype C HIV-1 vertical transmission patterns but further study to substantiate this finding is needed. To this end longitudinal samples will be extremely valuable to monitor the evolution of viral populations, and correlate the effect of genetic variation with disease progression and manifestation of clinical symptoms.

\section{ACKNOWLEDGMENTS}

This study was supported by PHS grants HD39620 and CA76958, Fogarty Training grant TW01429, and NCRR COBRE grant RR15635 to C.W. We thank Drs. James Hoxie and Weidong $\mathrm{Xu}$ for providing us with CCR5 32 cells and other reagents; Dr. Nathaniel Landau for HOS-CD4-CCR3 cells through the NIH AIDS Research and Reference Reagent Program, Division of AIDS; and Drs. John West and Evan Burkala for helpful discussion and review of our manuscript.

\section{SEQUENCE DATA}

GenBank accession numbers for 139 sequences in this article are AY135934-136071.

\section{REFERENCES}

1. Dickover RE, Garratty EM, Plaeger S, and Bryson YJ: Perinatal transmission of major, minor, and multiple maternal human immunodeficiency virus type 1 variants in utero and intrapartum. J Virol 2001;75:2194-2203.

2. Wolinsky SM, Wike CM, Korber BT, et al.: Selective transmission of human immunodeficiency virus type-1 variants from mothers to infants. Science 1992;255:1134-1137.

3. Lamers SL, Sleasman JW, She JX, et al:: Persistence of multiple maternal genotypes of human immunodeficiency virus type I in infants infected by vertical transmission. J Clin Invest 1994;93: 380-390.

4. Briant L, Wade CM, Puel J, Brown AJ, and Guyader M: Analysis of envelope sequence variants suggests multiple mechanisms of mother-to-child transmission of human immunodeficiency virus type 1. J Virol 1995;69:3778-3788.

5. Pasquier C, Cayrou C, Blancher A, et al.: Molecular evidence for mother-to-child transmission of multiple variants by analysis of RNA and DNA sequences of human immunodeficiency virus type 1. J Virol 1998;72:8493-8501.

6. Ndung'u T, Renjifo B, and Essex M: Construction and analysis of an infectious human immunodeficiency virus type 1 subtype $\mathrm{C}$ molecular clone. J Virol 2001;75:4964-4972.

7. Cecilia D, Kulkarni SS, Tripathy SP, Gangakhedkar RR, Paranjape RS, and Gadkari DA: Absence of coreceptor switch with disease progression in human immunodeficiency virus infections in India. Virology 2000;271:253-258.

8. Orti G, Pearse DE, and Avise JC: Phylogenetic assessment of length variation at a microsatellite locus. Proc Natl Acad Sci USA 1997;94:10745-10749.

9. Fu YX: Estimating effective population size or mutation rate using the frequencies of mutations of various classes in a sample of DNA sequences. Genetics 1994;138:1375-1386.

10. Meyerhans A, Cheynier R, Albert J, et al:: Temporal fluctuations in HIV quasispecies in vivo are not reflected by sequential HIV isolations. Cell 1989;58:901-910.

11. Kumar S, Tamura K, and Nei M: MEGA: Molecular Evolutionary Genetics Analysis, version 1. Pennsylvania State University, University Park, Pennsylvania, 1993.

12. Jiang S: HIV-1—co-receptors binding. Nat Med 1997;3:367-368.

13. Kim FM, Kolson DL, Balliet JW, Srinivasan A, and Collman RG: V3-independent determinants of macrophage tropism in a primary human immunodeficiency virus type 1 isolate. J Virol 1995;69: $1755-1761$

14. Fouchier RA, Groenink M, Kootstra NA, et al.: Phenotype-associated sequence variation in the third variable domain of the human immunodeficiency virus type 1 gp120 molecule. J Virol 1992;66:3183-3187.

15. Shioda T, Levy JA, and Cheng-Mayer C: Small amino acid changes in the V3 hypervariable region of gp120 can affect the T-cell-line and macrophage tropism of human immunodeficiency virus type 1. Proc Natl Acad Sci USA 1992;89:9434-9438. 
16. Xiao L, Owen SM, Goldman I, et al.: CCR5 coreceptor usage of non-syncytium-inducing primary HIV-1 is independent of phylogenetically distinct global HIV-1 isolaes: Delineation of consensus motif in the V3 domain that predicts CCR-5 usage. Virology 1998;240:83-92.

17. Oelrichs RB, Shrestha IL, Anderson DA, and Deacon NJ: The explosive human immunodeficiency virus type 1 epidemic among injecting drug users of Kathmandu, Nepal, is caused by a subtype C virus of restricted genetic diversity. J Virol 2000;74:1149-1157.

18. Reitter JN, Means RE, and Desrosiers RC: A role for carbohydrates in immune evasion in AIDS. Nat Med 1998;4:679-684.

19. Thompson JD, Gibson TJ, Plewniak F, Jeanmougin F, and Higgins DG: The CLUSTAL_X Windows interface: Flexible strategies for multiple sequence alignment aided by quality analysis tools. $\mathrm{Nu}-$ cleic Acids Res 1997;25:4876-4882.

20. Swofford DL: PAUP*: Phylogenetic Analysis Using Parsimony and Other Methods, version 4.0 beta. Sinauer Associates, Sunderland, Massachusetts, 1998.

21. Posada D and Crandall KA: MODELTEST: Testing the model of DNA substitution. Bioinformatics 1998;14:817-818.
22. Worobey M: A novel approach to detecting and measuring recombination: New insights into evolution in viruses, bacteria, and mitochondria. Mol Biol Evol 2001;18:1425-1434.

23. Rambaut A and Worobey M: PIST: Program for the InformativeSites Test, version 1. Department of Zoology, Oxford University, Oxford, 2001.

24. Sutthent R, Foongladda S, Chearskul S, et al.: V3 sequence diversity of HIV-1 subtype $\mathrm{E}$ in infected mothers and their infants. J Acquir Immune Defic Syndr Hum Retrovirol 1998;18:323-331.

Address reprint requests to:

Charles Wood

E318 Beadle Center

University of Nebraska

1901 Vine Street

Lincoln, Nebraska 68588-0666

E-mail: cwoodl@unl.edu 
This article has been cited by:

1. A. Ceballos, G. Andreani, C. Ripamonti, D. Dilernia, R. Mendez, R. D. Rabinovich, P. C. Cardenas, C. Zala, P. Cahn, G. Scarlatti, L. M. Peralta. 2008. Lack of viral selection in human immunodeficiency virus type 1 mother-to-child transmission with primary infection during late pregnancy and/or breastfeeding. Journal of General Virology 89:11, 2773-2782. [CrossRef]

2. Jessica D. Church, Wei Huang, Anthony Mwatha, Jonathan Toma, Eric Stawiski, Deborah Donnell, Laura A. Guay, Francis Mmiro, Philippa Musoke, J. Brooks Jackson, Neil Parkin, Susan H. Eshleman. 2008. HIV-1 Tropism and Survival in Vertically Infected Ugandan Infants. The Journal of Infectious Diseases 197:10, 1382-1388. [CrossRef]

3. Agn??s-Laurence Chenine, Kathleen A Buckley, Pei-Lin Li, Robert A Rasmussen, Helena Ong, Shisong Jiang, Tao Wang, Peter Augostini, W Evan Secor, Ruth M Ruprecht. 2005. Schistosoma mansoni infection promotes SHIV clade C replication in rhesus macaques. AIDS 19:16, 1793???1797. [CrossRef] 